\title{
A Rigorous Upper Bound of a Scalar Self-propagator in a Random Lattice Ensemble in Higher Dimensions
}

\author{
Hai-Cang Ren
}

The Institute for Advanced Study, Princeton, NJ 08540, USA

$$
\begin{aligned}
& \text { Abstract. Using an improved weight for a scalar field on a random lattice, it is } \\
& \text { rigorously proved that the self-propagator, averaged over an ensemble of } \\
& \text { random lattices with site density } \rho \text {, is bounded from above in } D \text { dimensions } \\
& (D>2) \text { i.e.: } \\
& \qquad \Delta_{0} \leqq\left(4+\frac{D}{2(D-2)} \omega_{D}^{2 / D-1} D !^{2 / D-1} D^{D / 2-1-2 / D}(D+1)^{D / 2-1 / 2-1 / D}\right) \\
& \qquad D^{D-2+2 / D} \omega_{D}^{2-2 / D} \Gamma\left(D-1+\frac{2}{D}\right) \rho^{1-2 / D},
\end{aligned}
$$

where $\omega_{D}$ is the solid angle in $D$ dimensions.

\section{Introduction}

One of the most intriguing problems in mathematical physics is the ultraviolet divergence. This problem arises from the uncontrollably vast fluctuation of the field configuration in the space-time continuum. By renormalization, one can get around this difficulty in a limited number of field theories. To deal with unrenormalizable theories, a formulation of discrete mechanics has been developed [1]. The basic assumption of this theory is: Within a finite space-time domain, say $\Omega$, one can only perform a finite number, say $\mathscr{N}$, of measurements on space time coordinates and field variables $\left(x_{n}, t_{n} ; \Phi_{n}\right)$. The weight of each measurement is $\exp \left(-A_{L}\right)$, where $A_{L}$ is the action of the field defined on a random lattice generated by $\left(x_{n}, t_{n}\right)$. Physical observables are obtained by averaging the ensemble of all measurements. Such a development invites a program of studying the effect in all branches of mathematical physics due to the discreteness of space-time. In this paper I pick up the simplest system of a free massless scalar field and focus on the finiteness of its ultraviolet behavior.

Originally such an attempt was made by Friedberg and Yancopoulos [2]. They succeeded in figuring out a rigorous upper bound of the scalar self-propagator in 
two dimensions based on the weight given in [3]. Their reasoning depends crucially upon the fact that the lattice scalar field action in two dimensions can be viewed as a continuum action with a piecewise linear field configuration plugged in. An improved definition of the weight proposed recently in [1] renders the above property true for the lattice action in all dimensions. Thus Friedberg's method can be generalized to higher dimensions. With some further technical modifications, the problem of bounding the self-propagator becomes tractable.

This paper is organized as follows: In Sect. II, properties of the improved weight for a scalar field will be reviewed and the problem will be formulated; in Sect. II, I will follow a series of steps similar to that in [2] to bound the self-propagator on an individual lattice; in Sect. IV, the ensemble average of the bound will be calculated.

\section{Formulation of the Problem}

Let $x_{i}(i=0, \ldots, \mathscr{N}-1)$ be a set of sites distributed arbitrarily within a $D$ dimensional Euclidean volume $\Omega$. Following [4] a unique rotationally invariant simplicial structure can be linked up by requiring that the inside of the circumsphere of each simplex be free of other lattice sites. We define a scalar field $\Phi_{i}$ at each site and the lattice action for the scalar field is given by:

$$
A_{L}=\frac{1}{2} \sum_{l_{l j}} \lambda_{i j}\left(\Phi_{i}-\Phi_{j}\right)^{2}
$$

where $l_{i j}$ is a link and $\lambda_{i j}$ is its weight. To determine $\lambda_{i j}$ we fill the inside of each simplex with a linear function $\Phi(x)$ such that $\Phi\left(x_{i}\right)=\Phi_{i}$, where $x_{i}$ is a vertex of the simplex. Then we define the lattice action to be the continuum action evaluated from this piecewise linear configuration, $\Phi(x)$, referred to later as the lattice configuration. The weight of the link $l_{i j}$ can be shown to be:

and

$$
\begin{gathered}
\lambda_{i j}=\left.\sum_{S} \lambda_{i j}(S)\right|_{l_{i j} \in S}, \\
\lambda_{i j}(S)=-\frac{1}{D^{2}} \frac{V_{i \mu} V_{j \mu}}{V},
\end{gathered}
$$

where $V$ is the volume of $S$, a $D$-simplex, and $V_{i \mu}$ is the volume, projected normal to the $\mu$-axis, of the $(D-1)$-simplex in $S$ opposite to site $i$. The orientation of $V_{i}$ is taken so that

$$
\sum_{i} V_{i \mu}=0 .
$$

It can be shown that the weight (2), (3) agrees with the weight defined in [3] in $D=2$ only.

A physical observable is given by the following ensemble average:

$$
\langle O\rangle=\frac{\int \Pi_{i=0}^{\mathcal{N}-1} d^{D} x_{i} J \int \Pi_{i=0}^{\mathcal{N}-1} d \Phi_{i} \exp \left(-A_{L}\right) O(\Phi)}{\int \Pi_{i=0}^{\mathcal{N}-1} d^{D} x_{i} J \int \Pi_{i=0}^{\mathcal{N}-1} d \Phi_{i} \exp \left(-A_{L}\right)},
$$

where $J$ is a Jacobian which has no effect on the continuum limit. The self- 
propagator at $p_{0}$ is given by

$$
\Delta_{0}=\frac{\int \Pi_{i=0}^{\mathcal{N}-1} d^{D} x_{i} J \int \Pi_{i=0}^{\mathcal{N}-1} d \Phi_{i} \exp \left(-A_{L}\right) \Phi_{0}^{2}}{\int \Pi_{i=0}^{\mathcal{N}-1} d^{D} x_{i} J \int \Pi_{i=0}^{\mathcal{N}-1} d \Phi_{i} \exp \left(-A_{L}\right)} .
$$

This expression is, however, not well defined because of the zero mode of $A_{L}$. To remove this ambiguity I specify the following boundary condition: Draw a large closed $(D-1)$-dimensional surface $\mathscr{B}$ whose shortest distance to $p_{0}$ is $R$ and $\rho^{1 / D}$ $R \gg 1$. We call a $(D-1)$-simplex an exterior simplex if its dual is severed by $\mathscr{B}$. The union of the exterior simplices forms a piecewise flat boundary $\mathscr{B}_{L}$ of the network. The potentials at the sites on $\mathscr{B}_{L}$ and outside it are fixed to be zero ${ }^{1}$. With this boundary condition we have:

$$
\begin{aligned}
\Delta_{0} & =\frac{\int \Pi_{i=0}^{\mathcal{N}-1} d^{D} x_{i} J \int \Pi_{i=0}^{N-1} d \Phi_{i} \exp \left(-A_{L}^{\prime}\right) \Phi_{0}^{2}}{\int \Pi_{i=0}^{\mathcal{N}-1} d^{D} x_{i} J \int \Pi_{i=0}^{N-1} d \Phi_{i} \exp \left(-A_{L}^{\prime}\right)} \\
& =\frac{\int \Pi_{i=0}^{\mathcal{N}-1} d^{D} x_{i} J(\operatorname{det} \mathscr{M})^{-1 / 2}\left(\mathscr{M}^{-1}\right)_{00}}{\int \Pi_{i=0}^{\mathcal{N}-1} d^{D} x_{i} J(\operatorname{det} \mathscr{M})^{-1 / 2}},
\end{aligned}
$$

where $A_{L}^{\prime} \equiv \frac{1}{2} \widetilde{\Phi} \mathscr{M} \Phi$ is the lattice action with the boundary condition plugged in and $N$, determined by the distribution of sites, is the total number of the sites whose potential are not fixed by the boundary condition. For the sake of simplicity we will take

Then

$$
\begin{aligned}
J & =(\operatorname{det} \mathscr{M})^{1 / 2}, \\
\Delta_{0} & =\frac{1}{\Omega^{\mathcal{N}}} \int \prod_{j=0}^{\mathcal{N}-1} d^{D} x_{j}\left(\mathscr{M}^{-1}\right)_{00} .
\end{aligned}
$$

The continuum limit of the self-propagator is:

$$
\Delta(0)=\frac{1}{(2 \pi)^{D}} \int d^{D} k \frac{1}{k^{2}},
$$

which is highly divergent. Intuitively we expect (9) to be finite. However the ensemble includes some lattices whose density near $p_{0}$ is very much greater than $\rho$ and $\left(\mathscr{M}^{-1}\right)_{00}$ will be correspondingly large, since the system acts like a continuum roughly down to the lattice spacing. As we shall see later the ensemble integration is indeed convergent. It follows from the positivity of $\mathscr{M}$ that $\left|\left(\mathscr{M}^{-1}\right)_{i j}\right| \leqq \frac{1}{2}\left(\left(\mathscr{M}^{-1}\right)_{i i}\right.$ $\left.+\left(\mathscr{M}^{-1}\right)_{j j}\right)$. Therefore, once the ensemble average of $\left(\mathscr{M}^{-1}\right)_{00}$ is bounded the ensemble averages of all other $\left(\mathscr{M}^{-1}\right)_{i j}(i \neq j)$ are also bounded.

\section{The Bound of the Self-propagator on an Individual Lattice}

The lattice scalar field system can be viewed as an electric network in multidimensional space. $\Phi_{i}$ can be identified as the voltage at each site, $\lambda_{i j}^{-1}$ as the resistance of each link and the action as the total power dissipated.

Let $p_{0}$ be the site on which the potential is fixed at $\Phi_{0}$ and the boundary

1 One can, of course, choose other boundary conditions. The ultraviolet behavior is not sensitive to the boundary condition 
condition be as given in the last section. By a variational principle it is easy to show that the absolute minimum of the power is given by

where

$$
P=\left(A_{L}^{\prime}\right)_{\min }=\frac{\Phi_{0}^{2}}{2 g}
$$

$$
g=\left(\mathscr{M}^{-1}\right)_{00} .
$$

Alternatively, we can also identify the power dissipated in the network as the power in a continuous conducting medium of unit conductivity evaluated from the lattice configuration and the boundary condition that $\Phi=0$ on $\mathscr{B}$ with the potential at $p_{0}$ being held at $\Phi\left(p_{0}\right)=\Phi_{0}$. We will take this point of view in what follows.

Theorem. For $g$ defined above we have:

where

$$
g \leqq g_{1}+g_{2}
$$

$$
\begin{gathered}
g_{1}=\left(\frac{4 R^{2}}{V}\right)_{\max }, \\
g_{2}=\frac{D}{2(D-2)} \omega_{D}^{2 / D-1} D !^{2 / D-1} D^{D / 2-1-2 / D}(D+1)^{D / 2-1 / 2-1 / D}\left(\frac{R^{2}}{V}\right)_{\max } .
\end{gathered}
$$

$\omega_{D}$ is the total solid angle in $D$ dimensions, $R$ is the circumradius of a simplex containing $p_{0}$ and $V$ is its volume. The minimum is taken among all the simplices containing $p_{0}$. Proof. To prove this theorem we subject the configuration to a series of modifications, each of which can only decrease or leave unchanged the total power dissipated. In fact most of the strategy follows [2], but some details are different because of the higher dimensionality.

We first define the intermediate polyhedron. It is the union of all the $D$-simplices containing $p_{0}$. The sites on this polyhedron except $p_{0}$ are called the intermediate sites and their potentials are called the intermediate potentials. The total power is then divided into two parts:

$$
P=P_{1}+P_{2},
$$

where $P_{1}$ is the power dissipated inside the intermediate polyhedron and $P_{2}$ is the power dissipated outside the intermediate polyhedron.

1) Let $p_{1}$ be the intermediate site with the lowest potential, $\Phi_{1}=\Phi\left(p_{1}\right), V_{1}$ be the volume of a simplex $S_{1}$ containing $\overline{p_{0} p_{1}}$ and $P\left(S_{1}\right)$ the power dissipated in $S_{1}$ only. Then:

$$
P_{1} \geqq P\left(S_{1}\right) \geqq \frac{1}{2}\left(\frac{\Phi_{0}-\Phi_{1}}{l}\right)^{2} V_{1} \geqq \frac{1}{2}\left(\frac{\Phi_{0}-\Phi_{1}}{2 R}\right)^{2} V_{1} \geqq \frac{\left(\Phi_{0}-\Phi_{1}\right)^{2}}{g_{1}},
$$

where

$$
g_{1}=4\left(\frac{R^{2}}{V}\right)_{\max } .
$$

2) We study $P_{2}$ by first lowering the potential on the intermediate polyhedron to its minimum $\Phi_{1}$. Then the power is decreased further. 
3) Relax the constraint of the lattice configuration and remove the boundary $\mathscr{B}$. We have then:

$$
P_{2} \geqq P_{c},
$$

where $P_{c}$ is the power dissipated in a conducting medium of an infinite size, when the intermediate polyhedron is held at constant potential $\Phi_{1}$.

4) Solving the Laplacian equation for a polyhedral boundary is awkward. In [2] the authors embedded a circle inside this polyhedron. The center of this circle is $p_{0}$ and the radius of the circle is the minimum altitude dropped from $p_{0}$ among all the simplices containing $p_{0}$. Then they fixed the potential on this circle at $\Phi_{1}$ and solved the Laplacian equation with the circular boundary. However this technique can not be generalized to dimensionality higher than 3 , as we shall see in the next section. The alternative I use is to pick up a simplex containing $p_{0}$ and embed an ellipsoid, referred to as the inner ellipsoid in what follows, completely inside this simplex. In Appendix A, we give a definition of such an ellipsoid. The volume of it is:

where

$$
\tau=(D-1) ! \omega_{D} D^{-D / 2}(D+1)^{-(D+1) / 2} V,
$$

$$
\omega_{D}=\frac{2 \pi^{D / 2}}{\Gamma\left(\frac{D}{2}\right)}
$$

is the total solid angle in $D$ dimensions and $V$ is the volume of the simplex chosen. Now the potential on the inner ellipsoid is fixed at $\Phi_{1}$. The power remains unchanged. Then we relax the potentials outside the ellipsoid. The power is decreased further.

5) In Appendix B we show that the power dissipated in a continuous medium by an ellipsoidal source at the potential $\Phi_{1}$ is given by:

$$
P_{c}=\frac{\omega_{D} \Phi_{1}^{2}}{\kappa}
$$

where

$$
\kappa=\int_{0}^{\infty} \frac{d s}{\sqrt{\left(s+a_{1}^{2}\right) \cdots\left(s+a_{D}^{2}\right)}},
$$

and $a_{1}, \ldots, a_{D}$ are the half-axes of the ellipsoid. $\kappa$ is a complicated integral and is bounded from above by:

$$
\kappa \leqq \frac{D}{D-2}\left(\frac{\omega_{D}}{D \tau}\right)^{1-2 / D},
$$

where $\tau$ is the volume of the ellipsoid. Using (18), (21), (22) and (23), we have:

$$
P_{2} \geqq P_{c} \geqq \frac{\Phi_{1}^{2}}{2 g_{2}},
$$

with $g_{2}$ given in (15). In deriving (24) we have used the inequality $\tau \leqq V \leqq V_{S}$, where $V$ is the volume of the simplex considered and $V_{S}$ is the volume of the circumsphere of the simplex. 
Combining (17) and (24) we obtain:

$$
P \geqq \frac{\left(\Phi_{0}-\Phi_{1}\right)^{2}}{2 g_{1}}+\frac{\Phi_{1}^{2}}{2 g_{2}} .
$$

Minimizing the right-hand side of (25) with respect to $\Phi_{1}$, we have

$$
P \geqq \frac{\Phi_{0}^{2}}{2\left(g_{1}+g_{2}\right)} \text {. }
$$

The theorem is proved.

From (12) we have the upper bound of the self-propagator for the individual lattice:

$$
\left(\mathscr{M}^{-1}\right)_{00} \leqq g_{1}+g_{2} .
$$

Therefore the ensemble average (5) is bounded by:

$$
\Delta_{0} \leqq\left\langle g_{1}\right\rangle+\left\langle g_{2}\right\rangle \text {. }
$$

\section{The Ensemble Average of the Upper Bound}

Both $g_{1}$ and $g_{2}$ are local quantities. Their ensemble averages can be easily bounded. Denote by $S_{a}, a=1, \ldots, m$ the $D$-simplices containing $p_{0}$, and by $R_{a}$ and $V_{a}$ the circumradius and the volumes of $S_{a}$. The probability density of $P\left(g_{1}\right)$ can be written as

$$
P\left(g_{1}\right)=\left\langle\delta\left(g_{1}-\max \left(\frac{4 R_{1}^{2}}{V_{1}}, \ldots, \frac{4 R_{m}^{2}}{V_{m}}\right)\right)\right\rangle,
$$

where $\langle\cdots\rangle$ means the ensemble average over random lattices. Define

Clearly

$$
\widetilde{P}\left(g_{1}\right)=\left\langle\sum_{a=1}^{m} \delta\left(g_{1}-\frac{4 R_{a}^{2}}{V_{a}}\right)\right\rangle .
$$

$$
\begin{aligned}
P\left(g_{1}\right) & \leqq \widetilde{P}\left(g_{1}\right), \\
\int d g_{1} P\left(g_{1}\right) & =1, \\
\int d g_{1} \widetilde{P}\left(g_{1}\right) & =N_{D / 0},
\end{aligned}
$$

where $N_{D / 0}$ is the average number of $D$-simplices containing a common site. Therefore

$$
\begin{aligned}
\left\langle g_{1}\right\rangle= & \int_{0}^{\infty} g_{1} P\left(g_{1}\right) d g_{1} \leqq \int_{0}^{\infty} g_{1} \tilde{P}\left(g_{1}\right) d g_{1} \\
= & \frac{1}{\Omega^{\mathcal{N}-1}} \int \prod_{i=1}^{\mathcal{N}-1} d^{D} x_{i} \sum_{a=1}^{m} \frac{4 R_{a}^{2}}{V_{a}}=\frac{1}{\Omega^{\mathscr{N}-1}} \int \prod_{i=1}^{\mathcal{N}-1} \\
& \cdot d^{D} x_{i} \sum_{x_{i_{1}} \ldots \ldots x_{i} D} f\left(x_{i_{1}}, \ldots, x_{i_{D}}\right) \frac{4 R^{2}\left(x_{i_{1}}, \ldots, x_{i_{D}}\right)}{V\left(x_{i_{1}}, \ldots, x_{i_{D}}\right)} \\
= & \frac{1}{\Omega^{\mathscr{N}-1}} \frac{(\mathscr{N}-1) !}{D !(\mathscr{N}-D-1) !} \int \prod_{i=1}^{\mathcal{N}-1} d^{D} x_{i} f\left(x_{1}, \ldots, x_{D}\right) \frac{4 R^{2}\left(x_{1}, \ldots, x_{D}\right)}{V\left(x_{1}, \ldots, x_{D}\right)}
\end{aligned}
$$


where

$$
f\left(x_{1}, \ldots, x_{D}\right)= \begin{cases}1 & \text { if } p_{0}, \ldots, p_{D} \text { form a simplex } \\ 0 & \text { otherwise }\end{cases}
$$

Let $p_{0}, p_{1}, \ldots, p_{D}$ be a $D$-simplex, $p_{C}$ be its circumcenter, $R$ be its circumradius and $V$ be its volume. The volume of its circumsphere is $v=\left(\omega_{D} / D\right) R^{D}$. Equation (34) can be written as

$$
\left\langle g_{1}\right\rangle \leqq \frac{1}{\Omega^{\mathcal{N}-1}} \frac{(\mathscr{N}-1) !}{D !(\mathscr{N}-D-1) !} \int d^{D} x_{1} \cdots d^{D} x_{D}(\Omega-v)^{\mathcal{N}-D-1} \frac{4 R^{2}}{V} .
$$

As $\mathscr{N} \rightarrow \infty$ with $\mathscr{N} / \Omega=\rho$ held fixed, we have

$$
\left\langle g_{1}\right\rangle=\frac{\rho^{D}}{D !} \int d^{D} x_{1} \cdots d^{D} x_{D} e^{-\rho v} \frac{4 R^{2}}{V} .
$$

Following (4), let us introduce a dummy point $x$ by inserting into (36) the following factor:

$$
1=\int d^{D} x \delta^{D}\left(x-x_{c}\right)=\int d^{D} x 2^{D} D ! V \delta\left(R_{0}^{2}-R_{1}^{2}\right) \cdots \delta\left(R_{0}^{2}-R_{D}^{2}\right)
$$

we have

$$
\begin{gathered}
\left\langle g_{1}\right\rangle=\frac{4 \rho^{D}}{D !} \int d^{D} x_{1} \cdots d^{D} x_{D} e^{-\left(\rho \omega_{D} / D\right) R^{D}} \frac{R^{2}}{V} \int d^{D} x \delta^{D}\left(x-x_{c}\right) \\
=2^{D+2} \rho^{D} \int d^{D} x_{0} \cdots d^{D} x_{D} e^{-\left(\rho \omega_{D} / D\right) R^{D}} R^{2} \delta\left(R_{0}^{2}-R_{1}^{2}\right) \cdots \delta\left(R_{0}^{2}-R_{D}^{2}\right)
\end{gathered}
$$

where $R_{i}=\overline{p_{i} p_{c}}$, and we have replaced $d^{D} x$ by $d^{D} x_{0}$. Introducing spherical coordinates $d^{D} x_{i}=R_{i}^{D-1} d R_{i} d \omega_{i}$, we have

$$
\begin{aligned}
\left\langle g_{1}\right\rangle & \leqq 4 \rho^{D} \int_{0}^{\infty} d R R^{(D-2)(D+1)+3} e^{-\left(\rho \omega_{D} / D\right) R^{D}} \omega_{D}^{D+1} \\
& =4 D^{D-2+2 / D} \omega_{D}^{2-2 / D} \Gamma\left(D-1+\frac{2}{D}\right) \rho^{1-2 / D} .
\end{aligned}
$$

From Eq. (15) we have

$$
\left\langle g_{2}\right\rangle=\frac{D}{D-2} \omega_{D}^{2 / D-1} D !^{2 / D-1} D^{D / 2-1-2 / D}(D+1)^{D / 2-1 / 2-1 / D} \frac{1}{8}\left\langle g_{1}\right\rangle .
$$

Therefore

$$
\begin{aligned}
\Delta_{0} \leqq & \left(4+\frac{D}{2(D-2)} \omega_{D}^{2 / D-1} D !^{2 / D-1} D^{D / 2-1-2 / D}(D+1)^{D / 2-1 / 2-1 / D}\right) \\
& \cdot D^{D-2+2 / D} \omega_{D}^{2-2 / D} \Gamma\left(D-1+\frac{2}{D}\right) \rho^{1-2 / D} .
\end{aligned}
$$

If we had used the method indicated in [2] to bound $g_{2}$, then $g_{2} \sim h^{2-D}$ with $h$ as the height of a simplex. However the measure of the ensemble average in (39) is $\sim h$ as $h$ small. Thus the integral would have diverged for $D>3$. This is avoided by using the inner ellipsoid. 
Table I. Numerical bound in various dimensions

\begin{tabular}{ll}
\hline$D$ & The bound \\
\hline 3 & $1.302 \times 10^{3} \rho^{1 / 3}$ \\
4 & $4.372 \times 10^{4} \rho^{1 / 2}$ \\
5 & $2.249 \times 10^{6} \rho^{3 / 5}$ \\
6 & $1.665 \times 10^{8} \rho^{2 / 3}$ \\
\hline
\end{tabular}

In Table I we list the numerical values of (42) in a few dimensions. It is not surprising that the bound is too high since only one of the simplices containing $p_{0}$ among many of them is picked up in bounding $P_{1}$ and $P_{2}$ (in $D=4$, say, there are about 160 simplices on the average containing a common site).

\section{Appendix A. The Inner Ellipsoid of a $D$-Simplex ${ }^{2}$}

Let $\overline{p_{0}, \ldots, p_{D}}$ form a $D$-simplex, $S_{D}$, in coordinate space $\left(x_{1}, \ldots, x_{D}\right)$, with $1_{0 i}$ as the links of $S_{D}$. By a linear transformation:

$$
x=\sum_{i=1}^{D} \zeta_{i} \mathbb{1}_{0 i}
$$

$S_{D}$ is deformed to a simplex $\mathscr{S}_{D}$ in coordinate space $\left(\zeta_{1}, \ldots, \zeta_{D}\right)$. The links $1_{01}, \ldots, 1_{0 D}$ of $\mathscr{S}_{D}$ have unit lengths and are perpendicular to each other. Let $\overline{p_{0}^{\prime}, \ldots, p_{D}^{\prime}}$ form a regular $D$-simplex, $S_{D}^{\prime}$, in coordinate space $\left(x_{1}^{\prime}, \ldots, x_{D}^{\prime}\right)$ with unit link length. By a linear transformation similar to (A1):

$$
x^{\prime}=\sum_{i=1}^{D} \zeta_{i} \mathbb{0}_{0 i}^{\prime}
$$

$S_{D}^{\prime}$ is deformed to the same $\mathscr{S}_{D}$. The combination of (A1) and the inverse of (A2) deforms $S_{D}$ to $S_{D}^{\prime}$. Since the transformation is linear, the volume ratio of two geometrical objects is invariant under such a deformation. Draw the inscribed sphere of $S_{D}^{\prime}$. The radius of this sphere is

and its volume is

$$
r_{D}^{\prime}=\sqrt{\frac{1}{2 D(D+1)}},
$$

$$
\tau_{D}^{\prime}=2^{-D / 2} \omega_{D} D^{-D / 2}(D+1)^{-D / 2} .
$$

The volume of the regular simplex is

$$
V_{D}^{\prime}=2^{-D / 2} \frac{\sqrt{D+1}}{D !}
$$

The volume ratio of this sphere and this regular simplex is

$$
\frac{\tau_{D}^{\prime}}{V_{D}^{\prime}}=(D-1) ! \omega_{D} D^{-D / 2}(D+1)^{-(D+1) / 2}
$$

2 I would like to thank R. Friedberg for suggesting this method of defining the inner ellipsoid 
When $S_{D}^{\prime}$ is deformed back to the original simplex, $S_{D}$, the inscribed sphere is deformed to an inscribed ellipsoid which we call the inner ellipsoid. From (A6), the volume of this ellipsoid is

$$
\tau_{D}=(D-1) ! \omega_{D} D^{-D / 2}(D+1)^{-(D+1) / 2} V_{D} .
$$

This gives (19) in the text.

\section{Appendix B. Ellipsoidal Coordinates in Arbitrary Dimensions}

Consider a family of $(D-1)$-dimensional quadratic surfaces in $D$-dimensional Euclidean space

$$
\sum_{i=1}^{D} \frac{x_{i}^{2}}{a_{i}^{2}+\theta}=1 ; \quad a_{1} \geqq \cdots \geqq a_{D},
$$

where $\theta$ is a parameter. Introduce a $D$ th-degree polynomial [5],

$$
f(\theta)=\left(a_{1}^{2}+\theta\right) \cdots\left(a_{D}^{2}+\theta\right)\left(1-\frac{x_{1}^{2}}{a_{1}^{2}+\theta} \cdots-\frac{x_{D}^{2}}{a_{D}^{2}+\theta}\right) .
$$

One can readily show that $f(\theta)$ has $D$ real roots in the following regions

$$
-a_{i}^{2} \leqq \zeta_{i} \leqq-a_{i+1}^{2} \quad\left(a_{D+1}^{2}=\infty\right)
$$

and

$$
f(\theta)=\left(\theta-\zeta_{1}\right) \cdots\left(\theta-\zeta_{D}\right) .
$$

Equations (B1) and (B2) give the transformation rule from the cartesian coordinates $\left(x_{1}, \ldots, x_{D}\right)$ to the ellipsoidal coordinates $\left(\zeta_{1}, \ldots, \zeta_{D}\right)$, i.e.

$$
x_{i}^{2}=(-1)^{D} \frac{\left(a_{i}^{2}+\zeta_{1}\right) \cdots\left(a_{i}^{2}+\zeta_{D}\right)}{\left(a_{1}^{2}-a_{i}^{2}\right) \cdots\left(a_{i-1}^{2}-a_{i}^{2}\right)\left(a_{i+1}^{2}-a_{i}^{2}\right) \cdots\left(a_{D}^{2}-a_{i}^{2}\right)} .
$$

It can be shown that $\left(\zeta_{1}, \ldots, \zeta_{D}\right)$ form a system of orthogonal coordinates and

where

$$
\sum_{i=1}^{D} d x_{i}^{2}=\sum_{i=1}^{D} \lambda_{i} d \zeta_{i}^{2}
$$

$$
\lambda_{i}=\frac{1\left(\zeta_{i}-\zeta_{1}\right) \cdots\left(\zeta_{i}-\zeta_{i+1}\right) \cdots\left(\zeta_{i}-\zeta_{D}\right)}{4} .
$$

The Laplacian equation in ellipsoidal coordinates is

$$
\sum_{i=1}^{D} \frac{1}{\sqrt{g}} \frac{\partial}{\partial \zeta_{i}}\left(\frac{\sqrt{g}}{\lambda_{i}} \frac{\partial}{\partial \zeta_{i}} \Phi\right)=0
$$

where

$$
g=\lambda_{1} \cdots \lambda_{D} .
$$

Consider an ellipsoid described by

$$
\sum_{i=1}^{D} \frac{x_{i}^{2}}{a_{i}^{2}}=1
$$


at constant potential $\Phi_{1}$, immersed in an infinite medium of unit conductivity. The potential in the medium will be a function of $\xi=\zeta_{D}$ only. In this case (B8) becomes

$$
\frac{d}{d \xi}\left(\sqrt{\left(a_{1}^{2}+\xi\right) \cdots\left(a_{D}^{2}+\xi\right)} \frac{d \Phi}{d \xi}\right)=0 .
$$

The solution satisfying $\Phi(0)=\Phi_{1}$ is

$$
\Phi(\xi)=\frac{\Phi_{1}}{\kappa} \int_{\xi}^{\infty} \frac{d s}{\sqrt{\left(a_{1}^{2}+s\right) \cdots\left(a_{D}^{2}+s\right)}}
$$

where $\kappa$ is given by

$$
\kappa=\int_{0}^{\infty} \frac{d s}{\sqrt{\left(a_{1}^{2}+s\right) \cdots\left(a_{D}^{2}+s\right)}} .
$$

The total power dissipated in the medium is

$$
P_{c}=\frac{1}{2} \int d^{D} x(\nabla \Phi)^{2}=\frac{\omega_{D} \Phi_{1}^{2}}{\kappa}
$$

Finally let us derive an upper bound of $\kappa$. Let $\delta$ be an arbitrary positive number. Then

$$
\begin{aligned}
\kappa & =\int_{0}^{\delta} \frac{d s}{\sqrt{\left(a_{1}^{2}+s\right) \cdots\left(a_{D}^{2}+s\right)}}+\int_{\delta}^{\infty} \frac{d s}{\sqrt{\left(a_{1}^{2}+s\right) \cdots\left(a_{D}^{2}+s\right)}} \\
& \leqq \frac{1}{a_{1} \cdots a_{D}} \delta+\int_{\delta}^{\infty} \frac{d s}{s^{D / 2}}=\frac{\omega_{D} \delta}{D \tau}+\frac{1}{\frac{D}{2}-1} \delta^{1-D / 2} .
\end{aligned}
$$

Minimizing the right-hand side of (B16) we obtain

$$
\kappa \leqq \frac{D}{D-2}\left(\frac{\omega_{D}}{D \tau}\right)^{1-2 / D}
$$

Acknowledgements. I am grateful to Professor R. Friedberg for critical reading of the manuscript. I am also indebted to Professor T. D. Lee for helpful discussions. This work was supported in part by the U.S. Department of Energy under contract number DE-AC02-76ER02220.

\section{References}

1. Lee, T. D.: Difference equations as the basis of fundamental physical theories, A talk given at the symposium, Old and New Problems in Fundamental Physics, held in honor of G. C. Wick in Pisa, Italy on Oct. 25 1984, and the references given there

2. Friedberg, R., Yancopoulos, S.: A rigorous upper bound in electrostatics on a random lattice ensemble. Commun. Math. Phys. 89, 131 (1983)

3. Christ, N. H., Friedberg, R., Lee, T. D.: Weights of links and plaquettes in a random lattice. Nucl. Phys. B210, 337 (1982) 
4. Christ, N. H., Friedberg, R., Lee, T. D.: Random lattice field theory: General formulation. Nucl. Phys. B202, 89 (1982)

5. Whittaker, E. T., Watson, G. N.: A course of modern analysis, fourth edition. Cambridge: Cambridge University Press 1940

Communicated by A. Jaffe

Received September 20, 1985; in revised form January 13, 1986 
